\title{
HYBRID OPTIMIZATION OF OBJECT-BASED CLASSIFICATION IN HIGH- RESOLUTION IMAGES USING CONTINOUS ANT COLONY ALGORITHM WITH EMPHASIS ON BUILDING DETECTION
}

\author{
E. Tamimi ${ }^{a *}$, H. Ebadi ${ }^{b}$, A. Kiani ${ }^{\mathrm{c}}$ \\ ${ }^{\text {a }}$ MS.c. Student, Faculty of Geodesy \& Geomatics Engineering, K.N.Toosi University of Technology, Tehran, Iran- \\ elahe.tamimi@gmail.com \\ ${ }^{\mathrm{b}}$ Prof., Faculty of Geodesy \& Geomatics Engineering, K.N.Toosi University of Technology, Tehran, Iran- \\ ebadi@kntu.ac.ir \\ ${ }^{c} \mathrm{PhD}$ student, Faculty of Geodesy \& Geomatics Engineering, K.N.Toosi University of Technology, Tehran, Iran- \\ abbasekiani@yahoo.com
}

\begin{abstract}
KEY WORDS: Hybrid Optimization, Object-based Classification, Ant Colony Optimization Algorithm, High Spatial Resolution Image.
\end{abstract}

\begin{abstract}
:
Automatic building detection from High Spatial Resolution (HSR) images is one of the most important issues in Remote Sensing (RS). Due to the limited number of spectral bands in HSR images, using other features will lead to improve accuracy. By adding these features, the presence probability of dependent features will be increased, which leads to accuracy reduction. In addition, some parameters should be determined in Support Vector Machine (SVM) classification. Therefore, it is necessary to simultaneously determine classification parameters and select independent features according to image type. Optimization algorithm is an efficient method to solve this problem. On the other hand, pixel-based classification faces several challenges such as producing salt-paper results and high computational time in high dimensional data. Hence, in this paper, a novel method is proposed to optimize objectbased SVM classification by applying continuous Ant Colony Optimization (ACO) algorithm. The advantages of the proposed method are relatively high automation level, independency of image scene and type, post processing reduction for building edge reconstruction and accuracy improvement. The proposed method was evaluated by pixel-based SVM and Random Forest (RF) classification in terms of accuracy. In comparison with optimized pixel-based SVM classification, the results showed that the proposed method improved quality factor and overall accuracy by $17 \%$ and $10 \%$, respectively. Also, in the proposed method, Kappa coefficient was improved by $6 \%$ rather than RF classification. Time processing of the proposed method was relatively low because of unit of image analysis (image object). These showed the superiority of the proposed method in terms of time and accuracy.
\end{abstract}

\section{INTRODUCTION}

Building detection by classifying Remote Sensing (RS) images has been attracted many researches' attention due to its extensive applications (e.g. automation of information extraction (Lari and Ebadi 2007, Hermosilla, Ruiz et al. 2011, $\mathrm{Li}, \mathrm{Wu}$ et al. 2013), updating Geographic Information System (GIS) database (Gharibi, Arefi et al. 2016) ,change detection and urban management (Bouziani, Goïta et al. 2010, Huang, Zhang et al. 2014)). In recent years, the development of RS sensors results in obtaining high spatial resolution (HSR) images. For this reason, it is possible to detect many features from these images, so the extracted information from these images are useful in urban management. Despite of the mentioned advantage, pixel-based classification in HSR images has faced many limitations (e.g. producing salt- paper results due to high spectral diversity, high time processing and inability to interpret image due to weakness of pixel information) (Chen, Hay et al. 2012). Therefore, with the development of objectbased techniques, the unit of image analysis changed from pixel to object (Blaschke 2010) and the limitations of pixel-based techniques have been solved (Hall and Hay 2003, Laliberte, Rango et al. 2004, Blaschke 2005, Desclée, Bogaert et al. 2006, Bontemps, Bogaert et al. 2008, Gamanya, De Maeyer et al. 2009). In object-based techniques, homogeneous image objects have more signal to noise ratio rather than image pixels. Therefore, the results obtained from object-based techniques are more accurate (Benz, Hofmann et al. 2004, Wang, Sousa et al.
2004, Ronczyk 2011, Petropoulos, Kalaitzidis et al. 2012). Since the input image is segmented into homogenous areas in object-based techniques, the earth's features are extracted based on reality. In other words, the main purpose of image processing is to extract features that match with reality (Castilla and Hay 2008).

There are two kinds of classification methods in terms of training samples availability (supervised and unsupervised) (Drăguț, Csillik et al. 2014, Chutia, Bhattacharyya et al. 2015, Egorov, Hansen et al. 2015) and image analysis unit (pixelbased and object-based) (Lillesand, Kiefer et al. 2014). Thorough different classification methods, many researchers suggested Support Vector Machine (SVM) as the superior method (Xuegong 2000, Camps-Valls, Gómez-Chova et al. 2004, Wang 2005, Khatami, Mountrakis et al. 2016). The advantages of SVM are simple training, good generalization ability (Li and Yin 2013, Bin, Jian et al. 2014, Cheng and Bao 2014, Ghamisi and Benediktsson 2015, Chen and Tian 2016) and efficiency in high dimensional and nonlinear problems with small samples (Gao, Mandal et al. 2010). SVM classification, as well as other methods, has some parameters (e.g. penalty term and kernel parameters). These parameters involve in training process of SVM and affect the classification performance, so these parameters values are essential to be determined before training process. The process of finding the appropriate parameter value is known as model selection (MS) or parameter optimization (Cheng and Bao 2014). There are many MS 
techniques such as grid search, gradient descent algorithm and trial and error approach. The limitations of these approaches are high time processing and low-level automation. On the other hand, building detection from HSR images has faced various limitations due to low number of spectral bands. For this reason, using other features according to the unit of image analysis (e.g. spectral, textural and geometrical features) $(\mathrm{Ji}$ 2000, Shackelford and Davis 2003, Coburn and Roberts 2004) or use of other data sources (e.g. digital elevation data) have been suggested to improve accuracy (Geerling, Labrador-Garcia et al. 2007). However, adding the additional features will lead to increase the presence probability of dependent features, which may reduce the accuracy. Therefore, it is essential to use some techniques to detect and remove dependent features, which leads to improve accuracy. This task is known as Feature Selection (FS) (Wan, Wang et al. 2016). Selecting appropriate features by trial and error approach is based on expert knowledge and is dependent on image type and scene. MS of SVM and also FS cause to apply continuous Ant Colony Optimization $\left(\mathrm{ACO}_{\mathrm{R}}\right)$ algorithm in order to solve the existence limitations. The advantages of $\mathrm{ACO}_{\mathrm{R}}$ are easy to realize and implement, parallel processing, suitable for continuous domain (Socha and Dorigo 2008, Zhang, Chen et al. 2010).

Many studies have been done in urban features detection based on image objects. For example, a comprehensive review of object-based features detection was presented by (Cheng and Han 2016). The limitations of the mentioned studies are lowautomation level, SVM parameter determination by trial and error approach (Petropoulos, Kalaitzidis et al. 2012, Liu and Bo 2015), using independent features according to expert knowledge (Liu and Bo 2015). For example, Bouziani in 2009 used only features which is selected by expert knowledge for building change detection. The limitations of the mentioned study was low automation level in FS procedure and the limited expert knowledge in all cases (Bouziani, Goïta et al. 2010). On the other hand, Samadzadegan et al. have optimized pixel-based SVM classification by using binary ACO with aim of FS and MS in hyperspectral imagery (Samadzadegan, Hasani et al. 2012, Samadzadegan, Hasani et al. 2012). MS of SVM is a continuous problem and FS is a discrete problem, so simultaneously optimization of SVM is a continuous problem. In the mentioned studies, they transformed continuous problem into discrete problem by discretizing continuous domain. On the other words, the continuous range of variable, which should be optimized, are converted into finite sets. This is not always appropriate, especially if the initial possible range of variable is wide (Socha and Dorigo 2008). In addition, the mentioned studies were based on pixel-based processing. As mentioned before, pixel-based processing result in higher time consuming in optimizing classification because of unit of image analysis (image pixel) and producing salt and paper result.

To overcome the above limitations and according to importance of selecting independent features in object-based classification, in this paper, a novel method is proposed with the aim of building detection based on hybrid optimization of object-based SVM classification in HSR image and elevation data. $\mathrm{ACO}_{\mathrm{R}}$ was applied because it is appropriate for continuous optimization problems. The advantages of the proposed method include independency of image scene, relatively high automation level, relatively high speed processing because of unit of image analysis (image object), decreasing the probability of post processing with the purpose of building edge reconstruction. The results of the proposed method were evaluated by pixel-based SVM classification in terms of time, Ground Truth (GT) in terms of accuracy and precision and Random Forest (RF) classification in terms of the ability of choosing independent features. The rest of this paper is organized as follows: the data are described in section 2 . The methods and the proposed method are illustrated in Section 3 and 4, respectively. The results are given in Section 5, together with the discussion. Conclusions are drawn in Section 6.

\section{DATA DESCRIPTION}

The proposed method has been applied on subset of the digital aerial image and Digital Surface Model (DSM) of Bandar Anzali, Gilan Province (North of Iran). The digital aerial image was acquired by Ultracam D with a spatial resolution of $0.08 \mathrm{~m}$ and 3-band multispectral imagery (Red, Green, and Blue) (Figure 1). A look at Figure 1 reveals that the studied area is related to a complex urban area.

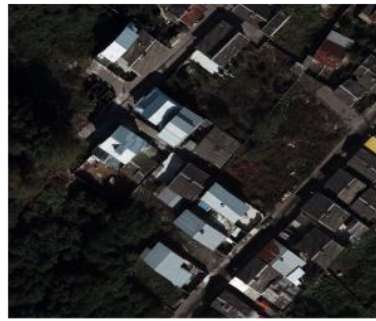

a. Digital aerial image

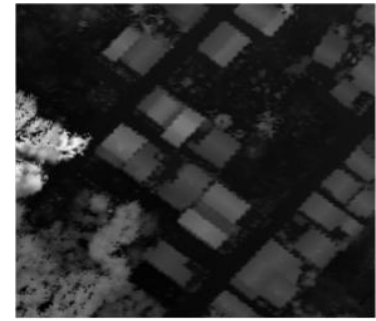

b. DSM
Figure 1. The study area: a. Digital Aerial image, b. DSM

\section{METHODS}

\subsection{Classification}

\subsubsection{Support Vector Machine Classification}

SVM, a supervised machine learning algorithm, was proposed by Vapnik (Vapnik and Vapnik 1998). SVM finds an optimal separating hyper plane that maximizes the margin between two classes. In nonlinear problems, data are not separable in the original feature space. In these cases, SVM uses kernel functions (e.g. linear, sigmoid, polynomial and Radial Basis Function (RBF)) in order to map data into higher dimensional space, so data can be separable in the new space (Figure 2). In this paper, RBF kernel function is used due to achieving good results in terms of accuracy and time processing (Luo, Zhou et al. 2002, Pal 2002, Zhang, Chen et al. 2010) (Equation 4).

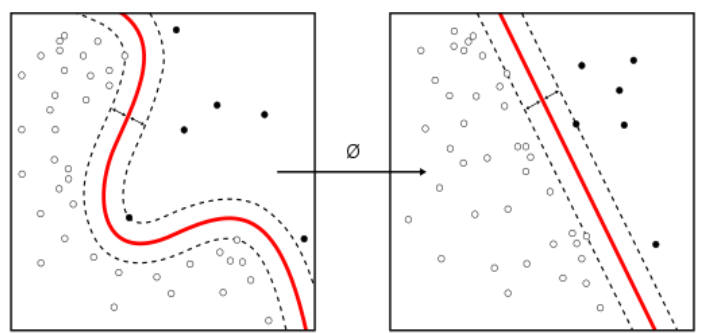

Figure 2. Map data from nonlinear space into higher dimensional space in SVM

Let consider a dataset with $\mathrm{n}$ samples $\left\{\left(x_{i}, y_{i}\right) \mid i=1, \ldots, n\right\}$, where $x_{i} \in \mathrm{R}^{k}$ is a feature vector and $y_{i} \in\{-1,1\}$ defines the label of $x_{i}$. The SVM finds a hyper plane in a high dimensional space that is able to separate the data with a maximum margin (Equation 1): 
$w^{t} \phi(x)+b=0$

In the above equation, $w$ is a weight vector, orthogonal to the hyper plan, $b$ is an offset term and $\phi$ is a mapping function. Maximizing the margin is equivalent to minimizing the norm of $w$. Thus, SVM is trained to solve the following minimization problem:

$$
\min \left(\frac{\left\|w^{2}\right\|}{2}+c \sum_{i=1}^{n} \xi_{i}\right)
$$

subject to: $y_{i}\left(w^{t} \phi\left(x_{i}\right)+b\right) \geq 1-\xi_{i}$

$\xi_{i} \geq 0 ; \forall i$

Where $\mathrm{C}$ is a regularization parameter that imposes a trade-off between the number of misclassification in the training data and the maximization of the margin and $\xi_{i}$ are stack variables. The decision function obtained through the solution of the minimization problem equation (3) is given by

$$
f(x)=\sum_{i=1}^{n s v} \alpha_{i} y_{i} \phi\left(x_{i}\right) \phi(x)+b
$$

Where the constants are called Lagrange multipliers determined in the minimization process; SV corresponds to the set of support vectors, training samples for which the associated Lagrange multipliers are larger than zero. The kernel function compute dot products between any pair of samples in the feature space.

$$
K_{R B F}\left(x_{i}, x_{j}\right)=\exp \left(-\frac{\left\|x_{i}-x_{j}\right\|}{2 \sigma^{2}}\right)
$$

\subsubsection{Random Forest Classification}

RF classification was proposed by Breiman in 2001. It is an ensemble classifier that apply a bootstrap aggregated sampling technique ("bagging") to build many individual decision trees, from which a final class assignment is determined (Breiman 2001). Observations in the original training data which do not occur in the bootstrap sample are named Out-Of-Bag (OOB) observations. A random subset of predictor variables split apart the training data into homogenous subsets (Mellor, Haywood et al. 2013). The node-splitting variable that allows for the greatest variance is selected. This allows the overall model to increase its generalization capacity before and after the split (Walton 2008). The OOB sample data evaluates the performance by computing the accuracy and error rates averaged over all of the predictions (Breiman 2001) and estimates the importance of each variable in the classification. In RF classification, it is possible to select dependent features by variable importance.

\subsection{3 $\mathrm{ACO}_{\mathrm{R}}$ Optimization Algorithm}

ACO was introduced as a novel nature-inspired method by (Dorigo, Maniezzo et al. 1996, Dorigo and Sttzle 2004). It is based on the foraging behaviour of real ant (finding the shortest path between their nest and food sources (optimization problem)). The real ants initially explore the area surrounding their nest in a random manner when searching for food. As soon as an ant finds a food source, it carries some food back to the nest and deposits a pheromone trail on the ground during the return trip. The amount of deposited pheromone shows the quantity and quality of the path between the food source and their nest and guides others ants to find the food source. After a while, the path that has the most pheromone is the shortest path between their nest and the food sources. Based on this, ant colonies choose this path as the shortest one. Indirect communication among ants via pheromone trails enables them to find the shortest path between their nest and food sources. This capability of real ant colonies has inspired the definition of artificial ant colonies that can find approximate solutions to optimization problems (Socha and Dorigo 2008).

Continuous optimization is hardly a new research field. ACO is suitable for discrete domain. For continuous domain, it was extended to continuous domains without making any major conceptual change to its structure. $\mathrm{ACO}_{\mathrm{R}}$ extended the population-based ACO with Gaussian Probability Density Function (PDF) as pheromone update and adopted the rankbased selection mechanism (Xiao and Li 2011). The advantages of $\mathrm{ACO}_{\mathrm{R}}$ is possible to solve problems where some variables are discrete and others are continuous (Socha and Dorigo 2008). $\mathrm{ACO}_{\mathrm{R}}$ uses initial population and the Gaussian PDFs in order to generate the best individuals of this population. After sampling from these Gaussian PDFs, the new individuals merge with the initial population. Finally, the predefined number of individual are selected from the new population sorted based on an objective function. The best solution is an individual that has the best objective function value in the stopping criterion. This procedure will continue until the stopping criterion is met. The general flowchart of $\mathrm{ACO}_{\mathrm{R}}$ is shown in Figure 3. Further details about $\mathrm{ACO}_{\mathrm{R}}$ can be found in (Socha and Dorigo 2008).

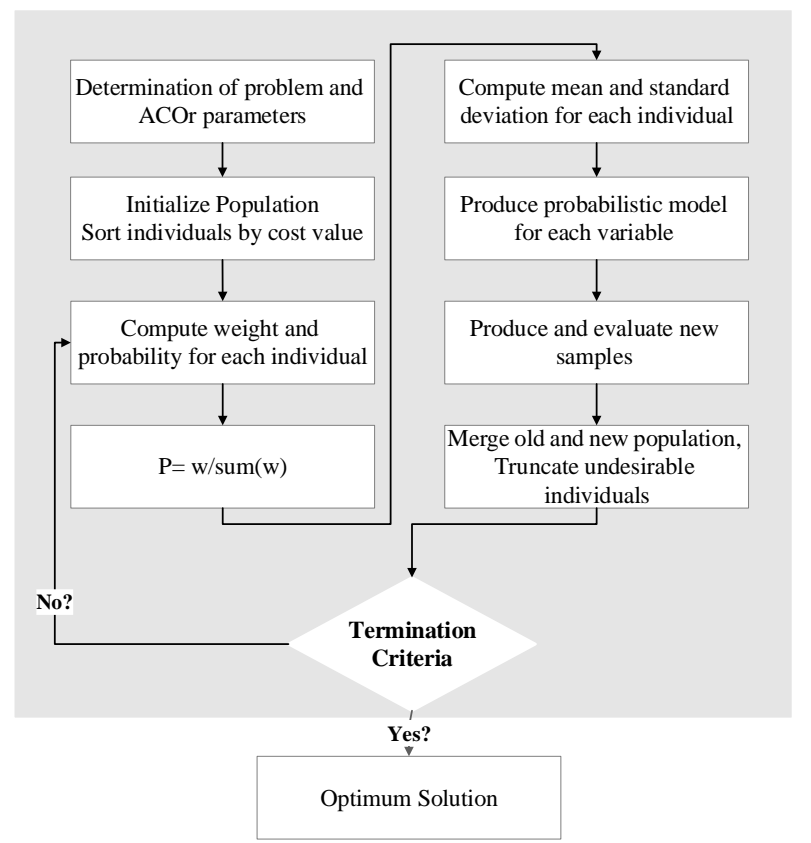

Figure 3. General Flowchart of $\mathrm{ACO}_{\mathrm{R}}$

\section{THE PROPOSED METHOD}

The main aim of this paper is to propose a new method to optimize object-based SVM classification in FS and MS. The proposed method includes six steps: image pre-processing, normalized Digital Surface Model (nDSM) generation, segmentation, feature extraction and preparation, hybrid optimization, object-based SVM classification by optimum results and accuracy assessment. The flowchart of the proposed method is shown in Figure 4, which is discussed in the following sections. 


\subsection{Image Pre-processing}

Image pre-processing (e.g. radiometric and geometric corrections) is the first step of most of the RS processing. The studied data sources were georefrenced. Histogram Equalization (HE) is a simple and effective radiometric correction method with the purpose of increasing contrast in images, which was applied on the image. It should be noted that the difference between the minimum and maximum value of brightness intensity (luminance) is low in image with low contrast. HE makes it possible to increase the contrast of input image. More details can be found in (Wang, Chen et al. 1999).

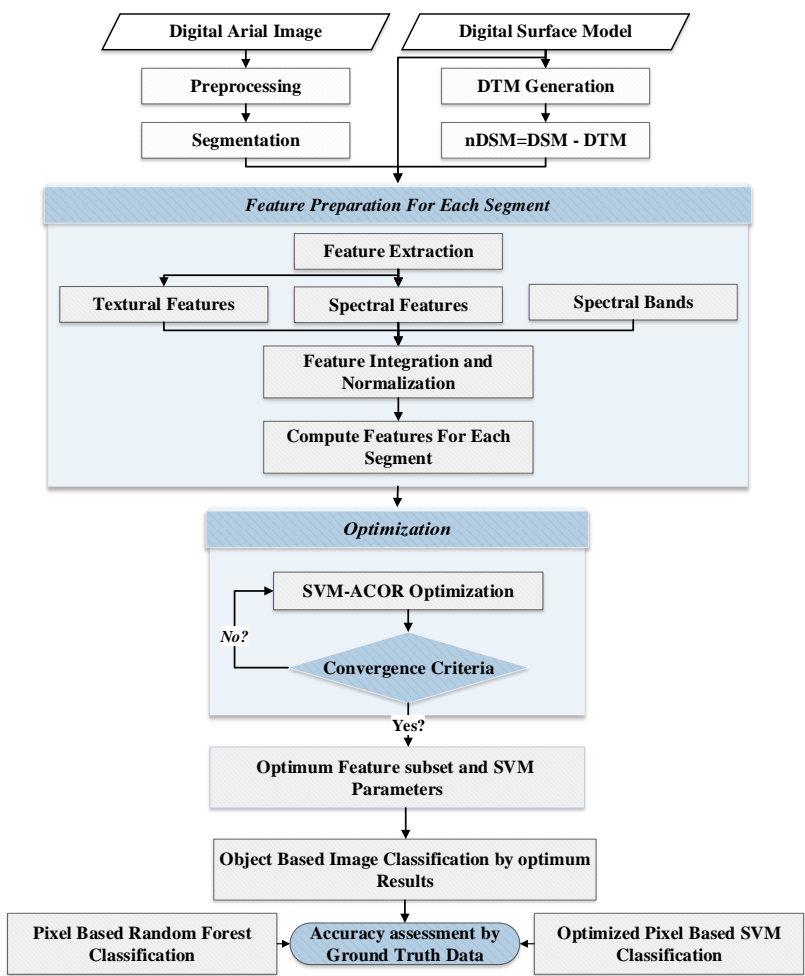

Figure 4. The flowchart of the proposed method

\subsection{Normalized Digital Surface Model Generation}

DSM and Digital Terrain Model (DTM) are essential to produce normalized DSM (nDSM). In order to identify the bare earth's surface and DTM generation, there are many filters which filter the ground and non-ground points. In this paper, Adaptive Triangulated Irregular Network Modelling (ATINM) was applied on DSM, which is capable of identifying the bare earth's points in complex urban areas. More detail about ATINM can be found in (Axelsson 2000). After that, DTM was produced by gridding the output results of applying ATINM on DSM using nearest neighbor method. Finally, nDSM was computed from Equation (5).

$$
n D S M=D S M-D T M
$$

\subsection{Image Segmentation}

Image segmentation is an important step in object-based analysis because its quality directly affects the success or failure of those processing based on the object-based analysis (Witharana and Civco 2014). It is the process of labelling pixels in an image such that all of the pixels located in a homogenous area have a similar label (Haris, Efstratiadis et al. 1998). Among the proposed segmentation algorithms, multi resolution segmentation was used in this paper. The advantages of this algorithm includes easy realizing and implementation and producing results that match reality (Baatz and Schäpe 2000, Lefebvre, Corpetti et al. 2008). This method consecutively merges pixels or existing image objects. It is a bottom-up segmentation based on a pairwise region merging technique. Multi resolution segmentation is an optimization procedure which, for a given number of image objects, minimizes the average heterogeneity and maximizes their respective homogeneity. More detail about this method can be found in (Baatz and Schäpe 2000, Lefebvre, Corpetti et al. 2008).

By applying multi resolution segmentation, the input image was segmented into homogenous areas. Since the proposed method was applied on the over segments, the segmentation parameters (i.e. scale, shape and compactness) were determined by trial and error approach. For this reason, it was not necessary to determine accurate segmentation parameters in order to create segments that math reality.

\subsection{Feature Extraction and Preparation}

Generally, there are three kinds of features: spectral, textural and structural. In this paper, spectral and textural features were extracted to appropriately separate different classes because the proposed method was applied on the over segments due to determination of the segmentation parameters by trial and error approach. According to (Barzegar, Ebadi et al. 2015), the spectral features used in this paper includes Excessive Green Index (EGI), Difference Green Ratio (DGR), Normalized Difference Index (NDI), Brightness Index (BI), Saturation Index (SaI), Hue Index (HI), Correlation Index (CI), Redness Index (RI), Shadow Index (SI) and LAB space (Table 1).

\begin{tabular}{|c|c|}
\hline Formula & Spectral Index \\
\hline $\begin{array}{l}\text { EGI (Woebbecke, Meyer et al. } \\
1995 \text { ) }\end{array}$ & $3 *((G) /(R+G+B))-1$ \\
\hline $\begin{array}{c}\text { DGR (Woebbecke, Meyer et } \\
\text { al. 1995) }\end{array}$ & $(G-R) /(R+G+B)$ \\
\hline $\begin{array}{c}\text { NDI (Woebbecke, Meyer et } \\
\text { al. 1995) }\end{array}$ & $(G-R) /(G+R)$ \\
\hline $\begin{array}{l}\text { BI (Mathieu, Pouget et al. } \\
\text { 1998) }\end{array}$ & $\left(\left(R^{2}+G^{2}+B^{2}\right) / 3\right)^{0.5}$ \\
\hline $\begin{array}{l}\text { SaI (Mathieu, Pouget et al. } \\
\text { 1998) }\end{array}$ & $(R-B) /(R+B)$ \\
\hline $\begin{array}{l}\text { HI (Mathieu, Pouget et al. } \\
\text { 1998) }\end{array}$ & $(2 * R-G-B) /(\mathrm{G}-\mathrm{B})$ \\
\hline $\begin{array}{l}\text { CI (Mathieu, Pouget et al. } \\
\text { 1998) }\end{array}$ & $(R-G) /(R+G)$ \\
\hline $\begin{array}{l}\text { RI (Mathieu, Pouget et al. } \\
\text { 1998) }\end{array}$ & $R^{2} /\left(B * G^{3}\right)$ \\
\hline SI & $(R+B+G) / 3$ \\
\hline
\end{tabular}

Table 1. The Spectral Features

The second type of features used in this paper were textural features which are extracted from Gray-Level Co-occurrence Matrix (GLCM). The idea of using GLCM was suggested by Haralick in the 1970s (Haralick, Shanmugam et al. 1973). Various textural features can be extracted from GLCM (e.g. mean, variance, homogeneity, contrast, dissimilarity, entropy, second moment, correlation). These features were extracted in five different kernel sizes (e.g. $3 \times 3,5 \times 5,7 \times 7,9 \times 9$ and $11 \times 11$ ) and four different directions (e.g. $0^{\circ}, 45^{\circ}, 90^{\circ}$ and $135^{\circ}$ ). Due to removing the impact of different directions on the textural features, the average of textural features was computed for each 
segment. Then all of the extracted features were integrated with spectral bands and the input image was normalized so that all image pixel values would be located in the range of $[0,1]$. After that, the extracted features were computed for each segment by averaging the values of all pixels located in each segment. This image was an input for the next step.

\subsection{Optimized Object-Based Classification}

\subsubsection{Hybrid Optimization}

Due to using various features and determination of SVM parameters, hybrid optimization was applied to select independent features and to determine the parameters of RBF kernel function $(\gamma)$ and penalty term $(C)$ with relatively high automation level. Based on this, it was essential to collect some training and test samples segments in order to train SVM and hybrid optimization. These samples were selected by an expert in two classes (building and non-building) with respect to GT data. In this paper, $\mathrm{ACO}_{\mathrm{R}}$ algorithm was used due to reasons discussed in section 1 and 3 .

In the initialize phase, the $\mathrm{ACO}_{\mathrm{R}}$ starts with an initial population. The initial population consisted of $\mathrm{N}$ individuals that were located in a D-dimensional search space. According to the optimization problem, each individual included three parts: mask of input features, $\mathrm{C}$ and $\gamma$. Thus, if the input image had (n) input features, the length of each possible solution will be $(\mathrm{n}+2)$. The $(\mathrm{n})$ first position in each possible solution are related to selecting that feature or not. The $(n+1)^{\text {th }}$ and $(n+2)^{\text {th }}$ positions were related to $\mathrm{C}$ and $\gamma$. After initializing individuals in the search space, each of them was evaluated by an objective function. In this paper, classification error was used as an objective function to evaluate each individual. An individual of the population was the best member that had the lowest classification error, so the objective of this optimization problem was to minimize the cost function. Finally, after reaching to the stopping criteria (i.e. maximum iteration) in $\mathrm{ACO}_{\mathrm{R}}$, the best individual with the lowest cost value was the optimum solution, which includes optimum features, $\mathrm{C}$ and $\gamma$.

\subsubsection{Object-Based SVM Classification}

After hybrid optimization, the object-based SVM classification was carried out by the optimum results. Then the classified image was evaluated by 5 different accuracy assessment criteria (e.g. Overall Accuracy (OA), Kappa Coefficient (KC), Quality Percentage (QP), Producer Accuracy (PA) and User Accuracy (UA)) with GT data. The results of the proposed method were also evaluated by pixel-based SVM and RF classification in terms of classification performance.

\section{RESULTS AND DISCUSSION}

The pre-processing procedures of the proposed method and the comparative methods were implemented using RS software (e.g. ALDPAT, Global Mapper, eCognition and ENVI) and MATLAB R2015a was used to implement the proposed method. To filter ground points from non-ground points, ATINM was applied on DSM in ALDPAT (Figure 5.a). The cell size of this filter was equal to the resolution of data sources $(0.08 \mathrm{~m})$. Then, DTM was produced by gridding the output results of applying ATINM on DSM using nearest neighbor method in Global Mapper. According to Equation 5, nDSM was computed (Figure 5.b).

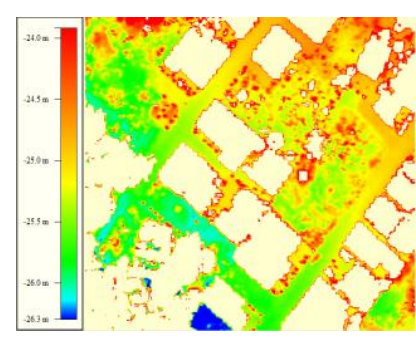

a. ATINM

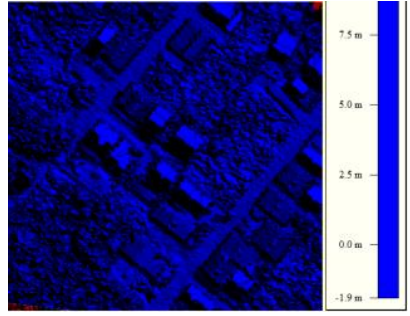

b. $\mathrm{nDSM}$
Figure 5.The result of a. ATINM, b. nDSM

As mentioned, it was essential to use HE method on the original image (Figure 6). After that, the spectral and textural features were extracted from HSR image and DSM in ENVI. Table 2 implies the characteristic these features.

\begin{tabular}{cc}
\hline \hline Feature & Number \\
\hline Spectral Feature & 12 \\
Initial Textural Feature & 640 \\
Final Textural Feature & 160 \\
Spectral Bands and nDSM & 4 \\
Total Feature & 176 \\
\hline \hline
\end{tabular}

Table 2. The characteristic of input features

Next, multi resolution segmentation was applied on the image in order to create homogenous areas in eCognition. The parameters of this segmentation are scale, shape and compactness. It is essential to set these parameters accurately with the purpose of exact feature extraction. However, in over segmentation procedure, these parameters were determined by default values according to image size, area type (e.g. urban or non-urban) and interest feature class (e.g. line features such as roads or regular features such as buildings). Based on this, the values of scale, shape and compactness parameters were set 60 , 0.8 and 0.3 , respectively. The segmented image is shown in Figure 7. After that, all of the extracted features were computed for each over segment by averaging all of the pixels located in each segment so the segmented image with the features was prepared for the hybrid optimization step.

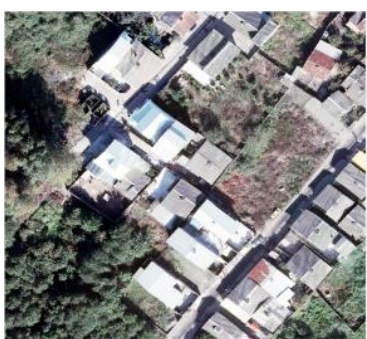

Figure 6 . The equalized image by HE method

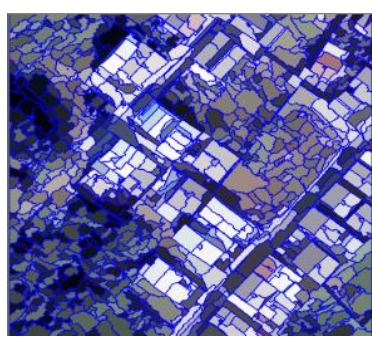

Figure 7. The over segmented image
In hybrid optimization step, it was essential to determine some parameters of optimization algorithm (e.g. number of individuals, maximum iteration, etc.). The number of individuals and maximum iteration in $\mathrm{ACO}_{\mathrm{R}}$ was 100 and 50, respectively. As stated in section 4, in hybrid optimization step, each individual of population was evaluated based on the objective function (cost function). In each iteration, each individual of population that had the lowest cost function was selected as an optimum solution in that iteration. The cost function of object-based SVM-ACOR and pixel-based SVMACOR were 4.651 and 35.509 in the first iteration, respectively 
and reduced in the last iteration. It showed that $\mathrm{ACO}_{\mathrm{R}}$ tries to find the best solution with the lowest cost function or the highest fitness function based on swarm intelligence. Since the objective function of this paper was classification error for training and test samples, the optimum solution was the solution with the lowest cost function (the best individual in the last iteration).

The improvement of KC, OA and QP for different classification methods is shown in Figure 8. According to Figure 8, QP, OA and $\mathrm{KC}$ were improved by $9 \%, 2 \%$ and $30 \%$ in object-based SVM classification using 15 randomly selected features (Object-SVM-15R) rather than using all of the input features (Object-SVM-176), respectively. It should be noted that SVM parameters were determined in trial and error approach in this step. This showed the presence probability of dependent features among all of the input features and their effects on classification performance. The accuracy criteria were improved by using 15 randomly selected features, but the improvement was not entirely appropriate. For this reason, it is essential to apply FS and MS methods in high dimensional space. In comparison with Object-SVM-15R, the proposed method (Object-SVM-ACOR improved QP, OA and KC by $20 \%, 12 \%$ and $30 \%$, respectively. These results showed the efficiency of the hybrid optimization in FS and MS procedure of SVM.

The optimized pixel-based SVM (Pixel-SVM-ACOR) reduced QP, OA and $\mathrm{KC}$ by $17 \%, 10 \%$ and $24 \%$ in comparison with Object-SVM-ACOR, respectively. These results showed the limitation of pixel-based classification in complex urban areas. Object-SVM-ACOR improved the $\mathrm{KC}$ by $6 \%$ rather than $\mathrm{RF}$ classification. According to good performance of RF in various RS studies, the relative improvement of the proposed method implied its superiority rather than RF in HSR images classification and clarified the efficiency of $\mathrm{ACO}_{\mathrm{R}}$ rather than other FS methods because $\mathrm{ACO}_{\mathrm{R}}$ is based on swarm intelligence that is more intelligent than other methods.

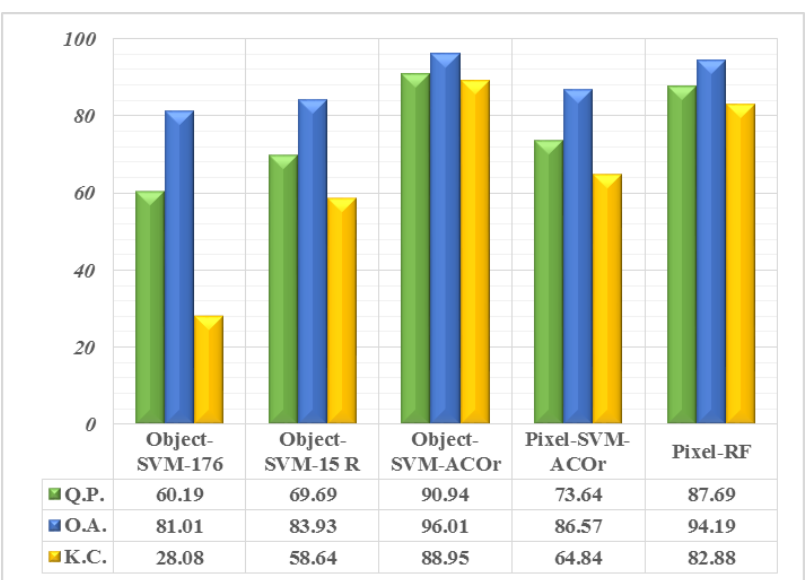

Figure 8. Chart of accuracy criteria in different methods

Different accuracy assessment criteria are shown in Table 3 for all of the methods. It should be noted that accuracy assessment was done by GT data. Time processing in the proposed method was relatively low in comparison with pixel-based methods. The reason of low time processing in the proposed method was the unit of image analysis (image object) because image objects reduced the computational complexity rather than pixels. While pixel was a unique unit in pixel-based techniques and this resulted in high time processing. The Object-SVM-ACOR provided more accurate results than pixel-based techniques. These showed the superiority of the proposed method rather than pixel-based techniques in terms of accuracy and time processing.

The classified images obtained from different methods are shown in Figure 9. A look at Figure 9 reveals that the proposed method had better performance than pixel-based classification in building detection and didn't result in salt-paper results that produced in RF classification. According to figure 9, some over segments of shadow on building roof were assigned to building class in the proposed method while these over segments were not classified in building class in pixel-based classification. This showed the limitation and weakness of pixel-based techniques. As it can be seen, some of the segments and also some pixels were misclassified into building class while they must be classified into non-building class (vegetation class). This was due to unavailability of Near Infrared band (NIR) in order to separate building class from non-building class (vegetation class). To address this problem, NIR band or spectral features based on this band (e.g. normalized vegetation index, etc.) or structural features or features extracted from LiDAR data (e.g. laplacian, slope) can be used. Due to applying the proposed method on the over segments and relatively high automation level, it was not possible to use structural features for each segment. It is suggested to apply optimization method in order to determine the segmentation parameter, then it will be possible to extract structural features in the classification process. In Figure 9, the buildings edges were identified clearer in Object-SVM-ACOR than RF. This resulted in post processing reduction for building edge reconstruction.

\begin{tabular}{|c|c|c|c|c|c|c|}
\hline & lod & Class & $\mathbf{U A}$ & $\mathbf{P A}$ & $\mathbf{Q P}$ & $\begin{array}{c}\text { Optimum } \\
\text { Features }\end{array}$ \\
\hline \multirow{9}{*}{ 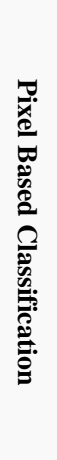 } & \multirow{3}{*}{$\begin{array}{l}\frac{\pi}{2} \\
\frac{1}{2} \\
\frac{1}{2}\end{array}$} & Building & 19.22 & 99.61 & 19.21 & \multirow{3}{*}{15} \\
\hline & & Non-Building & 99.97 & 79.84 & 99.97 & \\
\hline & & Total & 59.60 & 89.72 & 59.59 & \\
\hline & \multirow{3}{*}{ 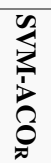 } & Building & 79.46 & 68.90 & 58.49 & \multirow{3}{*}{11} \\
\hline & & Non-Building & 88.79 & 93.26 & 88.79 & \\
\hline & & Total & 84.13 & 81.08 & 73.64 & \\
\hline & \multirow{3}{*}{$\pi$} & Building & 78.46 & 96.47 & 76.27 & \multirow{3}{*}{15} \\
\hline & & Non-Building & 99.10 & 93.64 & 99.10 & \\
\hline & & Total & 88.78 & 95.06 & 87.69 & \\
\hline \multirow{9}{*}{ 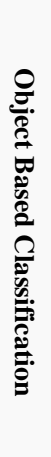 } & \multirow{3}{*}{ 荥 } & Building & 20.53 & 98.56 & 20.47 & \multirow{3}{*}{176} \\
\hline & & Non-Building & 99.91 & 80.09 & 99.91 & \\
\hline & & Total & 60.22 & 89.33 & 60.19 & \\
\hline & \multirow{3}{*}{ 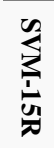 } & Building & 76.56 & 63.48 & 53.15 & \multirow{3}{*}{15} \\
\hline & & Non-Building & 86.23 & 92.17 & 86.24 & \\
\hline & & Total & 81.40 & 77.82 & 69.69 & \\
\hline & \multirow{3}{*}{$\begin{array}{l}3 \\
3 \\
3 \\
3 \\
3 \\
3\end{array}$} & Building & 91.47 & 91.69 & 84.47 & \multirow{3}{*}{12} \\
\hline & & Non-Building & 97.41 & 97.34 & 97.41 & \\
\hline & & Total & 94.44 & 94.51 & 90.94 & \\
\hline
\end{tabular}

Table 3. The accuracy assessment results obtained from different methods 


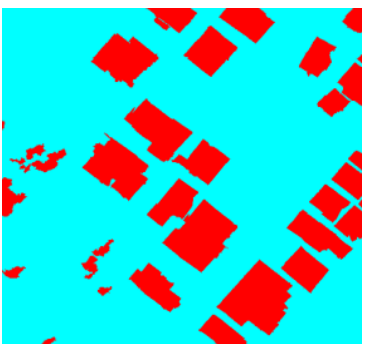

a.

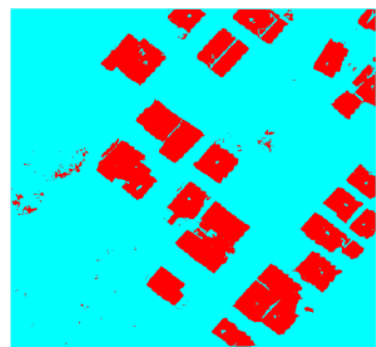

b.
Building

Non-Building

Figure 9. The Classified images obtianed from a. Object -SVM$\mathrm{ACO}_{\mathrm{R}}$ Clasification, b. RF Classification

\section{CONCLUSION}

Due to the existence limitation of pixel-based SVM classification in HSR images, object-based SVM classification was used in this paper. In nonlinear problems, it is essential to determine parameters of SVM because these parameters directly affect the results (MS). According to the limited number of spectral bands in HSR images, digital aerial images cannot provide information about classes with similar spectral behavior. Spectral and textural features or other data sources can be used to solve the limitations. However, adding some additional features will lead to increase the probability of dependent feature that may reduce the accuracy. Hybrid optimization of object-based SVM classification in MS of SVM and FS cause to apply ant colony optimization algorithm in order to solve the existence limitations. The advantages of the proposed method are relatively low time processing because of unit of image analysis, relatively high automation level, independency of image scene and type, post processing reduction for building edge reconstruction, hybrid optimization and accuracy improvement in comparison with pixel-based classification. The results showed that hybrid optimization of object-based SVM classification improved overall accuracy by $17 \%$ rather than object-based SVM by all of the input features. This showed the effect of hybrid optimization in classification performance and the presence probability of dependent features. Moreover, in comparison with hybrid optimization of pixelbased SVM classification, the overall accuracy and kappa coefficient were improved by $10 \%$ and $24 \%$ in the proposed method, respectively. This showed the superiority of optimized object-based SVM classification rather than optimized pixelbased SVM classification. Due to using over segments to satisfy automation level, there was no need to set fine values of the segmentation parameters that match reality. This resulted in lacking of structural features. For this reason, misclassification of some segments (misclassification of vegetation class into building class) were occurred. Therefore, it is recommended to propose a method to optimize the segmentation parameters, so structural features can be extracted for each segment in accordance with reality. Hence, the features can be used in classification that results in better separating between classes.

\section{REFERENCES}

Axelsson, P. (2000). "DEM generation from laser scanner data using adaptive TIN models." International Archives of
Photogrammetry and Remote Sensing 33(B4/1; PART 4): 111118.

Baatz, M. and A. Schäpe (2000). "Multiresolution segmentation: an optimization approach for high quality multiscale image segmentation." Angewandte Geographische Informationsverarbeitung XII 58: 12-23.

Barzegar, M., H. Ebadi and A. Kiani (2015). "Comparison of different vegetation indices for very high-resolution images, specific case UltraCam-D imagery." The International Archives of Photogrammetry, Remote Sensing and Spatial Information Sciences 40(1): 97.

Benz, U. C., P. Hofmann, G. Willhauck, I. Lingenfelder and M. Heynen (2004). "Multi-resolution, object-oriented fuzzy analysis of remote sensing data for GIS-ready information." ISPRS Journal of photogrammetry and remote sensing $\mathbf{5 8}(3)$ : 239-258.

Bin, W., Y. Jian, Z. Zhongming, M. Yu, Y. Anzhi, C. Jingbo, H. Dongxu, L. Xingchun and L. Shunxi (2014). "Parcel-Based Change Detection in Land-Use Maps by Adopting the Holistic Feature." Selected Topics in Applied Earth Observations and Remote Sensing, IEEE Journal of 7(8): 3482-3490.

Blaschke, T. (2005). "Towards a framework for change detection based on image objects." Göttinger Geographische Abhandlungen 113: 1-9.

Blaschke, T. (2010). "Object based image analysis for remote sensing." ISPRS journal of photogrammetry and remote sensing 65(1): 2-16.

Bontemps, S., P. Bogaert, N. Titeux and P. Defourny (2008). "An object-based change detection method accounting for temporal dependences in time series with medium to coarse spatial resolution." Remote Sensing of Environment 112(6): 3181-3191.

Bouziani, M., K. Goïta and D.-C. He (2010). "Automatic change detection of buildings in urban environment from very high spatial resolution images using existing geodatabase and prior knowledge." ISPRS Journal of Photogrammetry and Remote Sensing 65(1): 143-153.

Breiman, L. (2001). "Random forests." Machine learning 45(1): $5-32$.

Camps-Valls, G., L. Gómez-Chova, J. Calpe-Maravilla, J. D. Martín-Guerrero, E. Soria-Olivas, L. Alonso-Chordá and J. Moreno (2004). "Robust support vector method for hyperspectral data classification and knowledge discovery." IEEE Transactions on Geoscience and Remote sensing 42(7): 1530-1542.

Castilla, G. and G. Hay (2008). Image objects and geographic objects. Object-based image analysis, Springer: 91-110.

Chen, G., G. J. Hay, L. M. Carvalho and M. A. Wulder (2012). "Object-based change detection." International Journal of Remote Sensing 33(14): 4434-4457.

Chen, W. and Y. Tian (2016). "Parameter Optimization of SVM Based on Improved ACO for Data Classification." International Journal of Multimedia and Ubiquitous Engineering 11(1): 201212.

Cheng, G. and J. Han (2016). "A survey on object detection in optical remote sensing images." ISPRS Journal of Photogrammetry and Remote Sensing 117: 11-28.

Cheng, L. and W. Bao (2014). "Remote sensing image classification based on optimized support vector machine." Indonesian Journal of Electrical Engineering and Computer Science 12(2): 1037-1045.

Chutia, D., D. Bhattacharyya, K. Sarma, R. Kalita and S. Sudhakar (2015). "Hyperspectral remote sensing classifications: a perspective survey." Transactions in GIS. 
Coburn, C. and A. C. Roberts (2004). "A multiscale texture analysis procedure for improved forest stand classification." International journal of remote sensing 25(20): 4287-4308. Desclée, B., P. Bogaert and P. Defourny (2006). "Forest change detection by statistical object-based method." Remote Sensing of Environment 102(1): 1-11.

Dorigo, M., V. Maniezzo and A. Colorni (1996). "Ant system: optimization by a colony of cooperating agents." IEEE Transactions on Systems, Man, and Cybernetics, Part B (Cybernetics) 26(1): 29-41.

Dorigo, M. and T. Sttzle (2004). "Ant Colony OptimizationMIT Press." Cambridge, MA.

Drăguţ, L., O. Csillik, C. Eisank and D. Tiede (2014). "Automated parameterisation for multi-scale image segmentation on multiple layers." ISPRS Journal of Photogrammetry and Remote Sensing 88: 119-127.

Egorov, A., M. Hansen, D. Roy, A. Kommareddy and P. Potapov (2015). "Image interpretation-guided supervised classification using nested segmentation." Remote Sensing of Environment 165: 135-147.

Gamanya, R., P. De Maeyer and M. De Dapper (2009). "Objectoriented change detection for the city of Harare, Zimbabwe." Expert Systems with Applications 36(1): 571-588.

Gao, H., M. K. Mandal and J. Wan (2010). Classification of hyperspectral image with feature selection and parameter estimation. Measuring Technology and Mechatronics Automation (ICMTMA), 2010 International Conference on, IEEE.

Geerling, G., M. Labrador-Garcia, J. Clevers, A. Ragas and A. Smits (2007). "Classification of floodplain vegetation by data fusion of spectral (CASI) and LiDAR data." International Journal of Remote Sensing 28(19): 4263-4284.

Ghamisi, P. and J. A. Benediktsson (2015). "Feature selection based on hybridization of genetic algorithm and particle swarm optimization." Geoscience and Remote Sensing Letters, IEEE 12(2): 309-313.

Gharibi, M., H. Arefi, H. Rastiveis and H. Hashemi (2016). "Building Map Updating Based on Active Contour Models." Journal of Geomatics Science and Technology 5(4): 211-225.

Hall, O. and G. J. Hay (2003). "A multiscale object-specific approach to digital change detection." International Journal of Applied Earth Observation and Geoinformation 4(4): 311-327.

Haralick, R. M., K. Shanmugam and I. H. Dinstein (1973). "Textural features for image classification." Systems, Man and Cybernetics, IEEE Transactions on (6): 610-621.

Haris, K., S. N. Efstratiadis, N. Maglaveras and A. K. Katsaggelos (1998). "Hybrid image segmentation using watersheds and fast region merging." IEEE Transactions on image processing 7(12): 1684-1699.

Hermosilla, T., L. A. Ruiz, J. A. Recio and J. Estornell (2011). "Evaluation of automatic building detection approaches combining high resolution images and LiDAR data." Remote Sensing 3(6): 1188-1210.

Huang, X., L. Zhang and T. Zhu (2014). "Building change detection from multitemporal high-resolution remotely sensed images based on a morphological building index." Selected Topics in Applied Earth Observations and Remote Sensing, IEEE Journal of 7(1): 105-115.

Ji, C. (2000). "Land-use classification of remotely sensed data using Kohonen self-organizing feature map neural networks." Photogrammetric Engineering and Remote Sensing 66(12): 1451-1460.

Khatami, R., G. Mountrakis and S. V. Stehman (2016). "A meta-analysis of remote sensing research on supervised pixelbased land-cover image classification processes: General guidelines for practitioners and future research." Remote Sensing of Environment 177: 89-100.

Laliberte, A. S., A. Rango, K. M. Havstad, J. F. Paris, R. F. Beck, R. McNeely and A. L. Gonzalez (2004). "Object-oriented image analysis for mapping shrub encroachment from 1937 to 2003 in southern New Mexico." Remote Sensing of Environment 93(1): 198-210.

Lari, Z. and H. Ebadi (2007). Automatic extraction of building features from high resolution satellite images using artificial neural networks. Proceedings of ISPRS Conference on Information Extraction from SAR and Optical Data, with Emphasis on Developing Countries, Istanbul, Turkey.

Lefebvre, A., T. Corpetti and L. Hubert-Moy (2008). Objectoriented approach and texture analysis for change detection in very high resolution images. Geoscience and Remote Sensing Symposium, 2008. IGARSS 2008. IEEE International, IEEE. Li, C.-F. and J.-Y. Yin (2013). "Variational Bayesian independent component analysis-support vector machine for remote sensing classification." Computers \& Electrical Engineering 39(3): 717-726.

Li, Y., H. Wu, R. An, H. Xu, Q. He and J. Xu (2013). "An improved building boundary extraction algorithm based on fusion of optical imagery and LiDAR data." Optik-International Journal for Light and Electron Optics 124(22): 5357-5362.

Lillesand, T., R. W. Kiefer and J. Chipman (2014). Remote sensing and image interpretation, John Wiley \& Sons.

Liu, X. and Y. Bo (2015). "Object-based crop species classification based on the combination of airborne hyperspectral images and LiDAR data." Remote Sensing 7(1): 922-950.

Luo, J.-c., C.-h. Zhou, Y. Leung and J.-h. Ma (2002). "Support vector machine for spatial feature extraction and classification of remotely sensed imagery." JOURNAL OF REMOTE SENSING-BEIJING- 6(1): 55-61.

Mellor, A., A. Haywood, C. Stone and S. Jones (2013). "The performance of random forests in an operational setting for large area sclerophyll forest classification." Remote Sensing 5(6): 2838-2856.

Pal, M. (2002). Factors influencing the accuracy of remote sensing classifications: a comparative study, University of Nottingham.

Petropoulos, G. P., C. Kalaitzidis and K. P. Vadrevu (2012). "Support vector machines and object-based classification for obtaining land-use/cover cartography from Hyperion hyperspectral imagery." Computers \& Geosciences 41: 99-107. Ronczyk, M. (2011). "Object-based classification of urban land cover extraction using high spatial resolution imagery." URL: https://bismarck. nyme. hu.

Samadzadegan, F., H. Hasani and T. Schenk (2012). "Determination of optimum classifier and feature subset in hyperspectral images based on ant colony system." Photogrammetric Engineering \& Remote Sensing 78(12): 12611273.

Samadzadegan, F., H. Hasani and T. Schenk (2012). "Simultaneous feature selection and SVM parameter determination in classification of hyperspectral imagery using Ant Colony Optimization." Canadian Journal of Remote Sensing 38(2): 139-156.

Shackelford, A. K. and C. H. Davis (2003). "A hierarchical fuzzy classification approach for high-resolution multispectral data over urban areas." IEEE transactions on geoscience and remote sensing 41(9): 1920-1932.

Socha, K. and M. Dorigo (2008). "Ant colony optimization for continuous domains." European journal of operational research 185(3): $1155-1173$. 
Vapnik, V. N. and V. Vapnik (1998). Statistical learning theory, Wiley New York.

Walton, J. T. (2008). "Subpixel urban land cover estimation." Photogrammetric Engineering \& Remote Sensing 74(10): 12131222.

Wan, Y., M. Wang, Z. Ye and X. Lai (2016). "A feature selection method based on modified binary coded ant colony optimization algorithm." Applied Soft Computing 49: 248-258.

Wang, L. (2005). Support vector machines: theory and applications, Springer Science \& Business Media.

Wang, L., W. Sousa and P. Gong (2004). "Integration of objectbased and pixel-based classification for mapping mangroves with IKONOS imagery." International Journal of Remote Sensing 25(24): 5655-5668.

Wang, Y., Q. Chen and B. Zhang (1999). "Image enhancement based on equal area dualistic sub-image histogram equalization method." IEEE Transactions on Consumer Electronics 45(1): 68-75.

Witharana, C. and D. L. Civco (2014). "Optimizing multiresolution segmentation scale using empirical methods: Exploring the sensitivity of the supervised discrepancy measure Euclidean distance 2 (ED2)." ISPRS Journal of Photogrammetry and Remote Sensing 87: 108-121.

Woebbecke, D., G. Meyer, K. Von Bargen and D. Mortensen (1995). "Color indices for weed identification under various soil, residue, and lighting conditions." Transactions of the ASAE 38(1): 259-269.

Xiao, J. and L. Li (2011). "A hybrid ant colony optimization for continuous domains." Expert Systems with Applications 38(9): 11072-11077.

Xuegong, Z. (2000). "Introduction to statistical learning theory and support vector machines." Acta Automatica Sinica 26(1): $32-42$.

Zhang, X., X. Chen and Z. He (2010). "An ACO-based algorithm for parameter optimization of support vector machines." Expert Systems with Applications 37(9): 66186628 . 